\title{
Image Super-resolution with Improved Wiener Restoration and Simultaneous Edge Enhancement
}

\author{
Nisha. B.R \\ FISAT \\ Angamaly, Ernakulam \\ Kerala, India
}

\author{
Priya.S \\ Model Engineering College \\ Thrikkakara, Ernakulam \\ Kerala, India
}

\author{
Ashok Kumar. T \\ College of Engineering \\ Pallipuram, Cherthala \\ Kerala, India
}

\begin{abstract}
Super resolution is the process of increasing the spatial resolution of an image by exploiting additional sub pixel information from frames of input image sequences. Proposed method focuses on multi frame, spatial domain and reconstruction based super resolution techniques, namely, non uniform interpolation and iterated back projection. The performances of these two methods are improved by the addition of a restoration technique using improved Wiener restoration. Visual appearance of the image is further increased by iterative median filter that sharpens the edges. Results of the two methods are compared using a quality metric, peak signal to noise ratio (PSNR).
\end{abstract}

\section{General Terms}

Digital image processing, image pixel resolution, edge enhancement

\section{Keywords}

Super resolution, motion estimation, non uniform interpolation, iterated back projection, Wiener filter, iterative median filter

\section{INTRODUCTION}

Super resolution is the process of creating a high resolution (HR) image from multiple low resolution (LR) frames of an input sequence [1]-[4]. A high resolution image has not only high pixel density, i.e, more pixels per unit area of an image, but also more resolving power, which is very crucial for numerous applications, such as satellite and astronomy imaging, medical imaging, forensic imaging, microscopy, remote image sensing, video compression and processing etc. Images with more resolving power cannot be achieved merely by upsampling and interpolating the image. Such images may have more pixel density but may not have enough resolving power. High resolving power of an image makes the image details more clear. This is possible only by addition of high frequency components to the image, by suitable anti aliasing methods. Reduced spatial resolution of an image may be primarily due to hardware limitation of the imaging sensors that captures the image. Choice of an improved high resolution sensor is very expensive, and it is here the super resolution provides a cost effective solution. Super resolution (SR) techniques combine information from multiple low resolution frames that carries non redundant information pertaining to the same scene. The multiple LR frames that portray additional information of the same scene are possibly due to small camera movements or jitters, that exhibits sub pixel shifts between them. For super resolution, this relative displacement, i.e, sub pixel shifts, needs to be accurately estimated using some motion estimation algorithm and later on register them to a common reference grid. Generally, the low resolution frames of a scene is either obtained with different imaging sensors or taken over a period of time with a single sensor.

Broadly, the available super resolution techniques are classified based on three aspects 1) single frame super resolution and multi frame super resolution [5]-[6] 2) frequency domain super resolution and spatial domain super resolution [7]-[9] 3) reconstruction based super resolution and learning based super resolution [10]-[11]. Methods under these techniques have been extensively studied over the years. The proposed method combines the multi frame spatial domain and reconstruction based super resolution techniques that extracts additional sub pixel information from the input frames, register them and interpolates to a higher resolution grid followed with proper image restoration that undo the degradations of the image. First, simulation of low resolution frames are carried out by applying random warps in terms of translation, rotation and down sampling the image several times. Later on, the generated frames are registered using a spatial domain motion estimation method proposed by Keren et al. [12]. The high resolution image is reconstructed using two super resolution methods, namely non uniform interpolation and iterated back projection [13]. Finally restoration of the generated high resolution image is done using improved Wiener filter and iterative median filter that recovers the image from degradation and aliasing present, if any.

\subsection{Previous Works}

Super resolution of images has a history that stems back from 1980s when Huang and Tsay [7] developed and reconstructed the high resolution image using a frequency domain approach. This was based on the aliasing and shifting properties of continuous and discrete Fourier transform of the image. Drawback of this method is its inherent limitation to translation motion alone and linear space invariant blurs. The above limitations of frequency domain methods were later rectified by Vandewalle et al. [8]. Furthur, in 1982, Irani et al. [13] proposed iterated back projection method, which minimizes the error between the simulated LR images and observed ones by taking error back projection scheme into consideration. Stark and Oskoui [14] developed an algorithm based on projection onto convex sets (POCS), which addressed the reduction of blurring caused due to large pixels. Hardie et al. [15] proposed a steepest descent approach with block matching for super resolution while Chan et al. [16] proposed Tikhonov regularized super resolution approach. Spatial domain, reconstruction based approaches, which came later, could account for more realistic motion models and blurs. Capel et al. [17] conducted image registration with different methods and compared the performance of the 
generated high resolution image using maximum likelihood estimation (MLE) and maximum a posteriori (MAP) methods. Approach considering blur as a cue has also been proposed by Rajan et al. [18]. A wavelet based super resolution approach was proposed by $\mathrm{H} \mathrm{Ji}$ and $\mathrm{C}$ Fermuller [19]. Among the different survey reports on super resolution, the most prominent one is the technical survey done by Borman and Stevenson in 1998. It provides a complete and comprehensive study of different super resolution reconstruction methods along with directions for future advancements [20].

One of the crucial steps in multi frame super resolution technique is the motion estimation between the input low resolution frames [21]-[22]. Several motion estimation algorithms have already been proposed which can be mainly classified into frequency domain and spatial domain approaches. Some of the motion estimation methods under frequency domain are low frequency image matching method proposed by Vandewalle et al. [13], log polar based phase correlation method proposed by Marcel et al. [23] and a noise robust Cartesian coordinate frequency domain technique by Luchesse et al. [24]. The methods under spatial domain are random sample consensus (RANSAC) algorithm proposed by Fischler et al. [25], Taylor series based method proposed by Keren et al. [12] and several optical flow based methods [26][27]. Aliasing or degradations present in the generated high resolution image is removed using restoration techniques that improve the resolving power of the image and makes the image details more clear.

\subsection{Problem Formulation}

The process of super resolution is to improve the overall pixel density of an image and thereby improve the spatial resolution of the image. Pixel density is the amount of pixels that are present per unit area of an image. The first step in the super resolution process is the simulation of low resolution frames from the ground truth image. Generally, the image is subjected to different geometric warps like transrotational motion, blurs, contaminated with noise and later under sampled several times. This process creates several low resolution frames from which additional information is extracted using motion estimation. Each of the low resolution frames can be stated as

li $(x, y)=D \downarrow T i B i G(x, y)+N(x, y)$

where li is the generated $i$ th low resolution frame, Ti is the geometric warp, $\mathrm{Bi}$ is the blur function, $\mathrm{G}(\mathrm{x}, \mathrm{y})$ is the ground truth image and $\mathrm{N}(\mathrm{x}, \mathrm{y})$ is the additive noise. $\downarrow$ is the down sampling operator. Alternately, the mathematical model can be written as

$L i=Y i G+N$

where $\mathrm{Li}$ represents each of the low resolution images, $\mathrm{G}$ is the ground truth image, $\mathrm{Yi}$ is the combined effect of $\mathrm{Ti}, \mathrm{Bi}$ and $\mathrm{D}$ of equation 1 and $\mathrm{N}$ is the additive noise. The inverse problem of super resolution is to thus find an estimate of the original image $\mathrm{G}$ having higher spatial resolution, from $\mathrm{Li}, \mathrm{Yi}$ and $\mathrm{N}$.

\section{PROPOSED METHOD}

The proposed work starts with a simulation of low resolution images from the original ground truth image by applying random warps on the original ground truth image and down sampling them. The low resolution images are generated such that they exhibit sub pixel shift displacement between them. These sub pixel shifts contain additional information on the scene that can be acquired using apt motion estimation algorithms. The proposed method makes use of the method by Keren et al. [12], which is a spatial domain motion estimation approach. Later the simulated low resolution images and the motion estimates are passed to super resolution algorithm based on non uniform interpolation and iterated back projection. The resultant high resolution image is subjected to restoration using improved Wiener filter and iterative median filter for removing blur and noise present, if any. An overall block diagram of the proposed method is shown in figure 1.

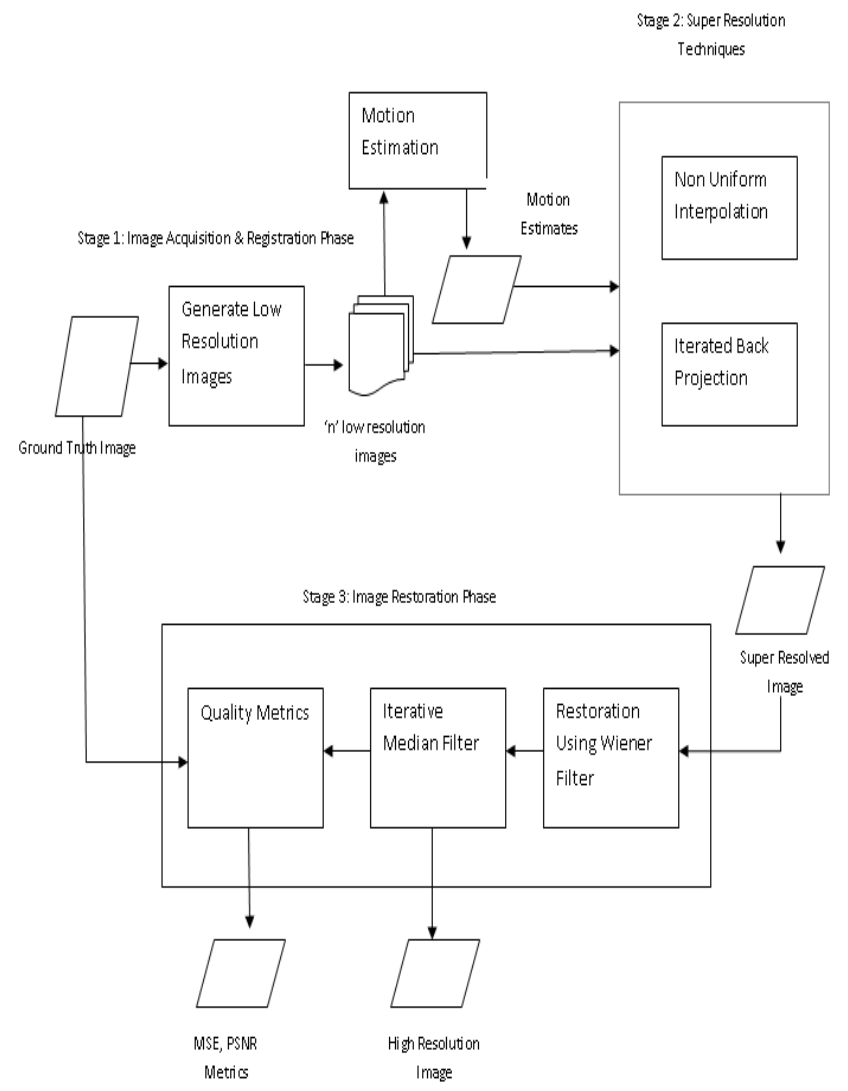

Figure 1: Proposed Method Block Schematic

\subsection{Motion Estimation}

The quality of the generated high resolution image heavily depends on the accuracy of the motion estimation method. Incorrect registration of low resolution frames results in degradation of the quality of the generated high resolution image. The proposed method adopts a spatial domain approach by Keren et al. [12] for motion estimation that outperforms the frequency domain methods by considering a transrotational motion model. The approach by Keren et al. [12] is a planar motion estimation method based on Taylor series expansion. It uses an iterative pyramidal scheme to increase the precision for larger motion parameters. The proposed method considers only planar motion between the low resolution frames. Also this method works best when the motions between the frames are small. A significant advantage is that since only small motions are considered, higher order terms in the Taylor series can be discarded, reducing the number of computations. Moreover, the proposed method accounts for both translation and rotation warps, unlike the conventional super resolution approaches, which take translation shifts only. The accuracy of the motion estimation is further increased by warping the frames back to normal using their initial 
computed rotation estimates. Although this method is time consuming, the accuracy of the final motion makes up for it.

Apart from this, the reference frame, which is the very first frame of the input sequence, is kept unchanged during the entire motion estimation process. Then the motion estimation between the reference frame and all other frames in the input sequence is carried out. This reduces the number of calculations and thus speeds up the overall process. Moreover, a Gaussian pyramidal structure is assumed, where the lower pyramidal images are considered first. i.e. motion estimates of lowest pyramidal frames are calculated first and then moved over to the higher ones.

\subsection{SR Algorithms}

The super resolution approaches considered in the proposed work are non uniform interpolation and iterated back projection, and their performances are compared. Since the low resolution frames are simulated using arbitrary translation and rotation warps, the registered images after motion estimation will be non uniformly distributed. A non uniform interpolation algorithm maps those pixels to a uniform high resolution grid using suitable interpolation methods. Out of the different interpolation methods like nearest neighbor interpolation, bilinear interpolation, B spline interpolation, bicubic interpolation etc, the proposed method adopts bicubic interpolation. The inherent demerit of this method is that it introduces more blur artifacts over the edges. Also, the performance of this method heavily depends on the accuracy of motion estimates.

Iterated back projection method proposed by Irani et al. [13] proceeds by estimating the error or difference between the observed low resolution images and simulated ones and updates the estimation of this displacement iteratively. This method also takes into consideration the blur using the point spread function (PSF). Initially a rough estimate of the high resolution image is made, and the process continues with the calculation of error accumulated between the original low resolution images and the simulated low resolution images. The error is compensated by adding it to the image gradient. A rough estimate of the original image is found by up sampling one of the low resolution images. The simulated low resolution images are generated from this up sampled image. The error accumulated is corrected using a back projection factor that makes the process more efficient. Demerits of this method are the over dependence of the result on arbitrary values of back projection factor and the absence of regularization. Due to this the algorithms converges to more than one solution. But unlike the non uniform interpolation method, the resulting high resolution image suffers from less edge artifacts and blurs.

\subsection{Improved Wiener Restoration}

Visual enhancement of the output image can be done by proper restoration. One of the main task of any restoration method is to undo the degradation occurred on the original image. The proposed method uses Wiener filter for the restoration of the generated HR image. Wiener filter is an optimal non linear filter, which is based on the concept of least squares and is a classical solution to minimize the mean square error (MSE), which is essentially the difference between the original image and the restored image [28]. It operates on the Fourier domain of the image. The advantage of Wiener filter is that it can handle both degradation as well as noise. Normally, Wiener filter works best when the point spread function of the blurring function is known. But this is not always true. In real situations, the cause of blur or degradation will be practically unknown and an efficient filter must be able to cope with such situations. A general Wiener filter may be stated as:

$F(u, v)=1 / H(u, v)\left\{\frac{\left|H(u, v)^{2}\right|}{\left|H(u, v)^{2}\right|+\frac{s_{\eta}(u, v)}{s_{f}(u, v)}}\right\} G(u, v)(3)$

where $\mathrm{H}$ is the Fourier transform of the point spread function, $\mathrm{S}_{\eta}(\mathrm{u}, \mathrm{v})$ and $\mathrm{S}_{\mathrm{f}}(\mathrm{u}, \mathrm{v})$ are the power spectrum of noise and the image respectively. To obtain an optimal Wiener filter result, it is essential to acquire correct knowledge of the covariance of the ground truth image. For accurate estimation of the covariance factor, a set of sample images of the ground truth image is usually taken. But in real situations, this may not be possible, and perhaps only a single version of degraded image will be available for the estimation of covariance. The proposed method makes use of an improved Weiner restoration, where the known PSF factor $\frac{S_{\eta}(u, v)}{S_{f}(u, v)}$ is replaced with a constant $\mathrm{X}$. Thus the equation can be rewritten as:

$F(u, v)=1 / H(u, v)\left\{\frac{\left|H(u, v)^{2}\right|}{\left|H(u, v)^{2}\right|+X}\right\} G(u, v)$

Where, $\mathrm{X}$ is a weighting function that is given with an initial value of 0.001 . For each iteration of Wiener restoration that follows, the value of $\mathrm{X}$ is incremented by 0.1 to a maximum threshold value. Apart from this, an error value is calculated and the process is repeated many times until the error value falls below a particular threshold. The $X$ value that minimizes the error value and makes it fall below the threshold is chosen as the ideal value for $\mathrm{X}$. This ideal value is used for final Wiener restoration. The performance of Wiener filter is significantly increased due to this iterative approach

\subsection{Iterative Median Filter}

Median filters are statistical non linear filters that are described in the spatial domain. Median filter performs smoothening of the image by computing the median of the neighborhood pixels of the image. The concept of median filter was introduced by Tukey in 1977 and its extension to two dimensional images was discussed by Pratt in 1978. A conventional median filter operates on every pixel of the image irrespective of the noise content, which makes it a time consuming operation. Over the years, numerous advancements in median filtering were introduced, some of which are switched median filtering, weighted median filtering, adaptive median filtering, iterative median filtering, median filters with fuzzy logic etc. Median filter performs the following task to find each pixel value in the processed image.

All pixels in the neighborhood of the pixels in the original image which are identified by the mask are sorted in either ascending or descending order.

The median of the sorted value of the pixels is computed and is chosen as the pixel value for the processed image.

For edge enhancement in the proposed method, a non linear edge preserving and smoothing filter such as iterative weighted median filter is used [29]-[31]. In the case of iterative median filter, if $y=f(x)$, where $x$ is the input, and $y$ is the output image, then median filtering is performed iteratively until the input image becomes approximately equal to the output image, i.e. $\mathrm{x}$ approximately equals to $\mathrm{y}$. The iterative weighted median filter has weights associated with each of the filter element, making it more efficient. 


\section{EXPERIMENTS}

Experiments were conducted on a variety of grayscale images and the results of the two super resolution algorithms were compared using a quality metric, peak signal to noise ratio.

For simulation of low resolution frames, the input image was subjected to small random shifts, in both horizontal and vertical direction and in small rotation angles, later on it is down sampled by factor of 4 in each direction. The number of low resolution images generated were set to 4 . No external blur or noise was applied to the generated low resolution frames. For the super resolution algorithms non uniform interpolation and iterated back projection, magnification factor was set to 4 . In Iterated Back Projection $\lambda$ factor was set to 0.1 . The constant $X$ value in Wiener filter was initially set as 0.001 , and later on incremented by 0.1 until a threshold value of 5 was reached. Iterative weighted median filter was repeated 10 times for edge sharpening.

Figure 2 shows the low resolution frames of the original image crow, each having a resolution [97x130]. The low resolution frames were created by subjecting the ground truth image to small translation shifts and rotation angles and down sampling by a factor of 4 in both horizontal and vertical directions.

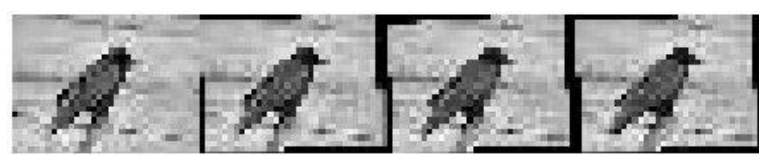

Figure 2: Crow: Low resolution frames

Figure 3a) and 3b) shows the high resolution (HR) result generated by the super resolution algorithms, non uniform interpolation and iterated back projection respectively. Images are super resolved by a magnification factor of 4 and the resulting high resolution image has a resolution of [388x520]. Figure $3 \mathrm{a}$ ) shows the result of non uniform interpolation (NUI) and $3 b$ ) shows the result of iterated back projection (IBP). It can be seen that the super resolved images $3 a$ ) and $3 b$ ), obtained after non uniform interpolation and iterated back projection, exhibits blur along the edges of the image. Non uniform interpolated image in figure $3 a$ ) exhibits more artifacts compared to that of figure $3 b$ ).

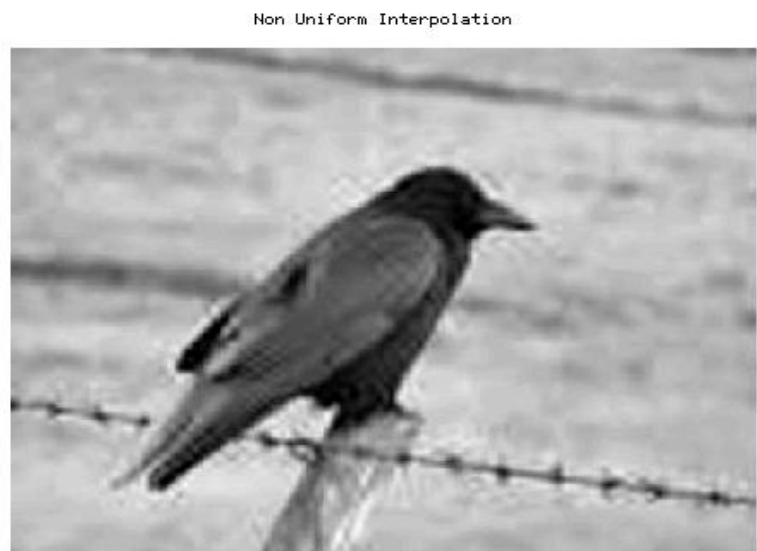

Figure 3a) Non Uniform Interpolation

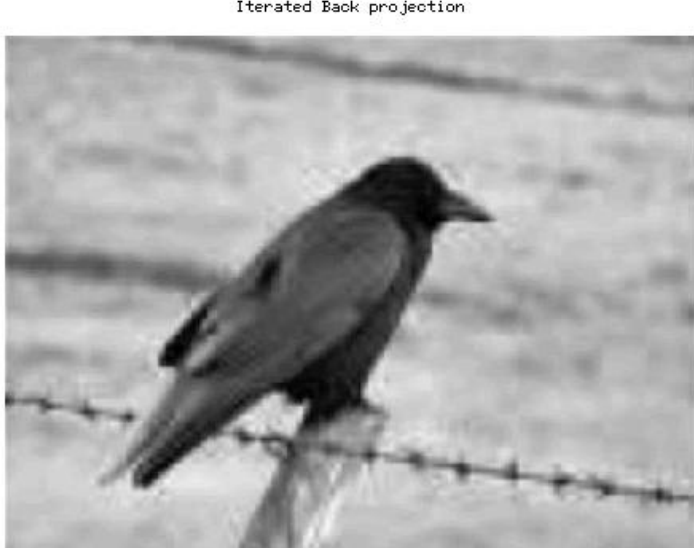

Figure 3b) Iterated back Projection.

Figure 4a) and $4 \mathrm{~b}$ ) shows the two high resolution (HR) results subjected to improved Wiener restoration. Figure 4a) shows the effect of improved Wiener restoration in the non uniform interpolated HR image and Figure 4b) shows the effect of improved Wiener restoration in the iterated back projected HR image.

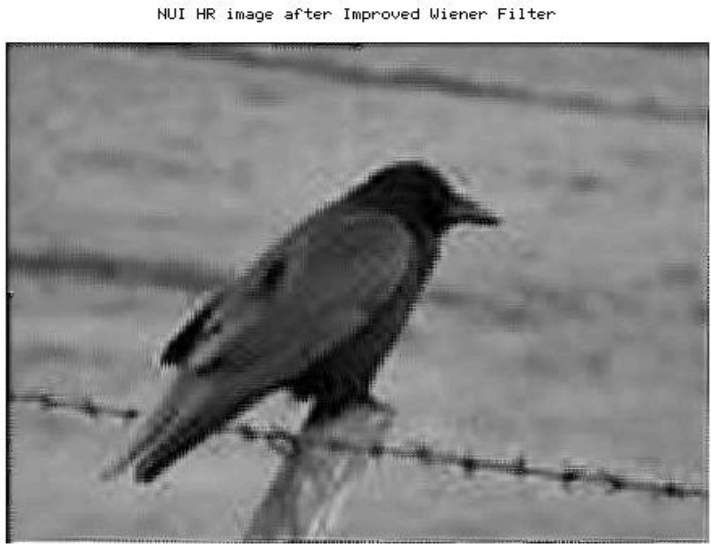

Figure 4a) NUI HR Image after Improved Wiener Restoration.

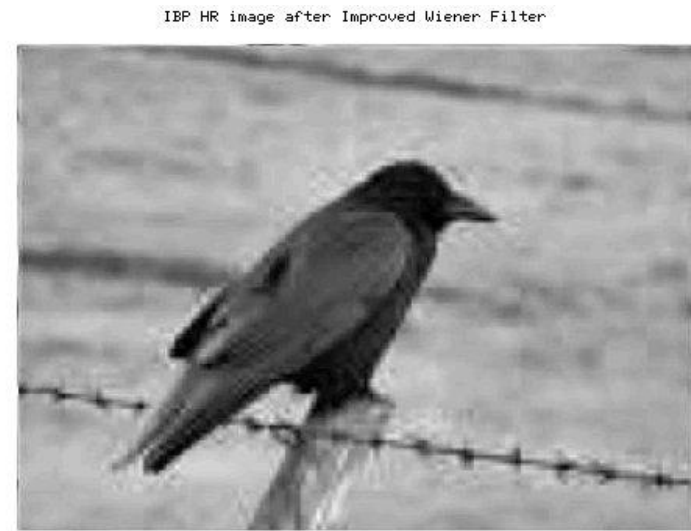

Figure 4b) IBP HR Image after Improved Wiener Restoration.

Figure 5a) and 5b) shows the two high resolution (HR) results after Improved Wiener restoration subjected to iterative median filter. Figure 5a) shows the effect of iterative median filter in the non uniform interpolated, improved Wiener restored HR image and Figure 5b) shows the effect of iterative median filter in the 
iterated back projected improved Wiener restored HR image. It can be seen that the images are free from any blur or aliasing and has achieved significant visual enhancement.

NUI Improved Wiener restored image after Iterative Median Filter

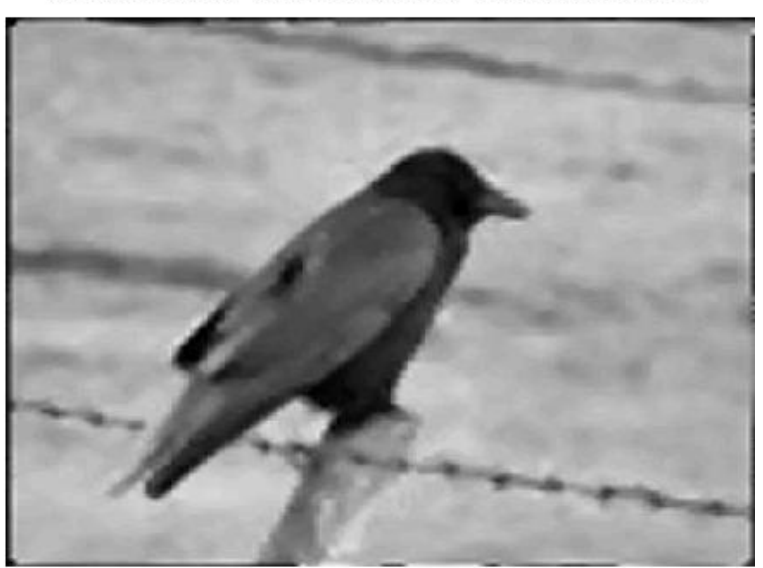

Figure 5a) NUI Improved Wiener restored HR Image after Iterative Median Filter.

The proposed method was implemented in QtOctave, an open source mathematical library similar to MATLAB, used for numerical computations, and was tested on a variety of gray scale input images. The simulated low resolution images generated from different ground truth images are shown in figure $6 a)-6 f)$. Quality of the obtained high resolution images were calculated using peak signal to noise ratio (PSNR) metric.

Peak signal to noise ratio is the ratio between the highest possible power of a signal to the power of the noise corrupting the signal that affects the fidelity of the signal. Logarithmic decibel $(\mathrm{dB})$ scale is used to express the value of PSNR. PSNR may be defined as

$$
\operatorname{PSNR}(x, y)=10 \log _{10}\left\{\frac{I_{\max }^{2}}{\operatorname{MSE}(x, y)}\right\}
$$

where $I_{\max }=255$ and MSE is defined as

$$
\operatorname{MSE}=\frac{1}{m n} \sum_{i j}\{x(i, j)-y(i, j)\}^{2}
$$

Table 1 shows the PSNR metrics of both high resolution images generated using non uniform interpolation and iterated back projection, followed by restoration. Figure 6a) - 6f) shows the different input low resolution frame sequences.

Figure 6(a) - 6(f) shows the different input low resolution frame sequences. The PSNR metrics of the generated HR images from these inputs are compared in Table 1.
IBP Improved Wiener restoredHR image after Iterative Median Filter

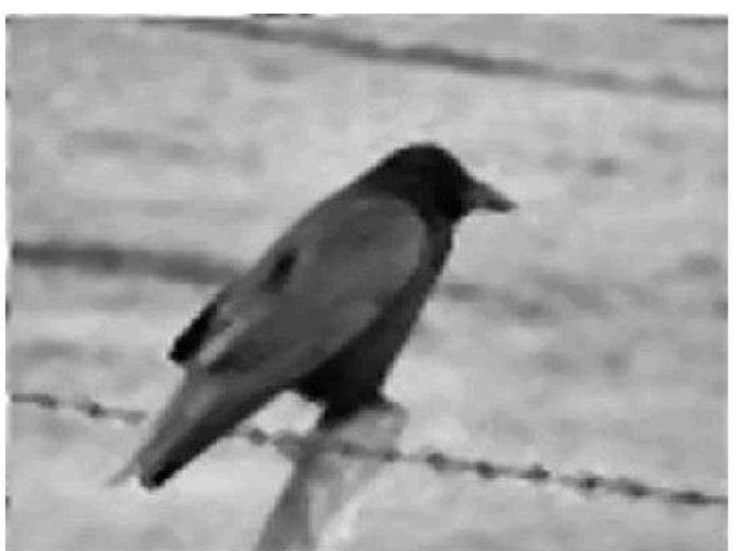

Figure 5b) IBP Improved Wiener restored HR Image after Iterative Median Filter.

Baby 1

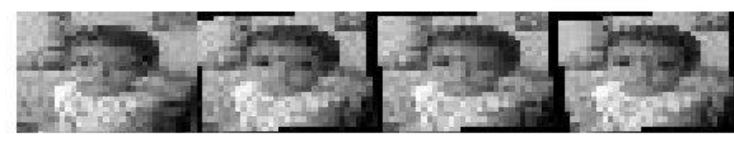

Fig 6a) baby 1

Baby 2

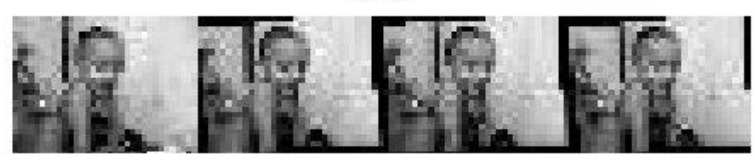

Fig 6b) baby 2

Baby 3

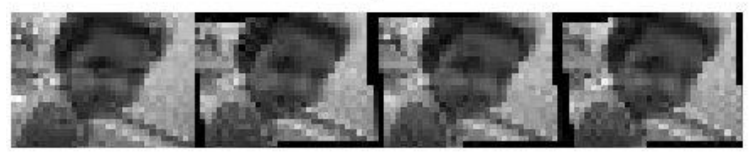

Fig 6c) baby 3

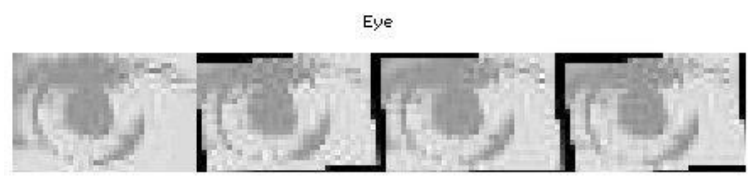

Fig 6d) eye

JackFruit

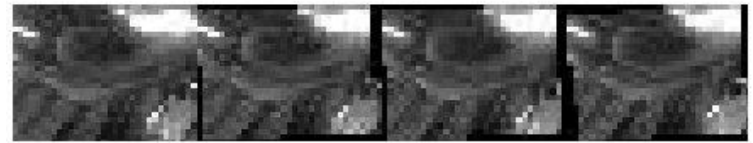

Fig 6e) jackfruit

Portrait

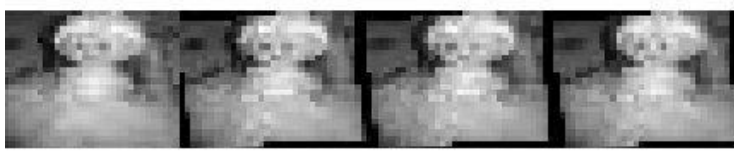

Fig 6f) portrait 
Figure 7a)-7c) shows the result of image baby2. Figure 7a) shows plain zoomed version of the input image, where the resolution is [97x130]. This image exhibits a lot of degradations with diminished picture quality. However Figure 7(b) and 7(c) shows the super resolved final high resolution result after restoration from non uniform interpolation and iterated back projection respectively. Resolution has been increased to [388x520]. It is evident that apart from increase in resolution, degradations are also removed with significant visible enhancement of the image.

\section{Zooned baby2}

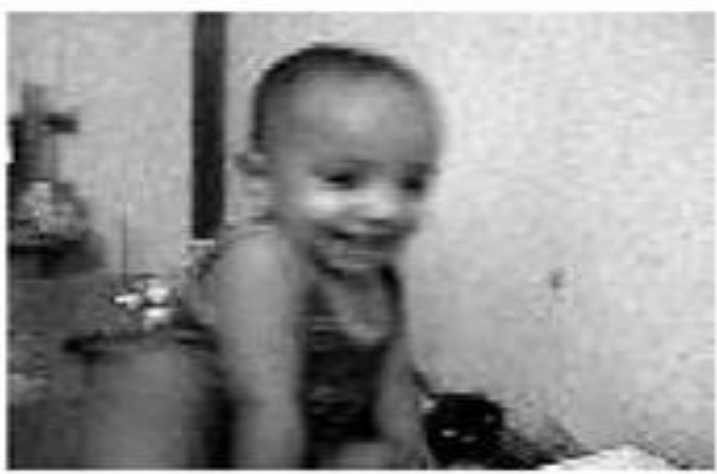

Figure 7a) Zoomed version of baby 2

Super resolution NJI HR

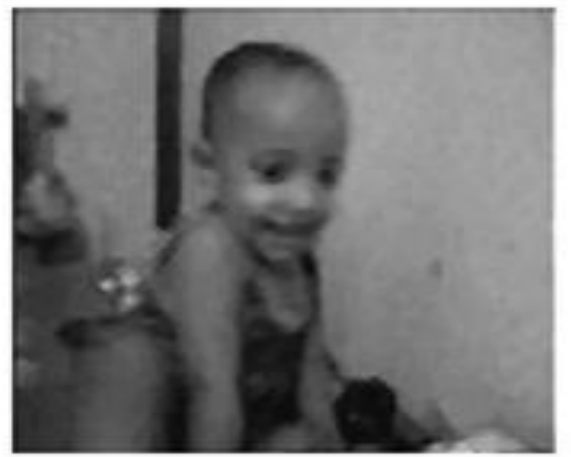

Figure 7b) HR image after NUI and image restoration

Super resolution IBP He

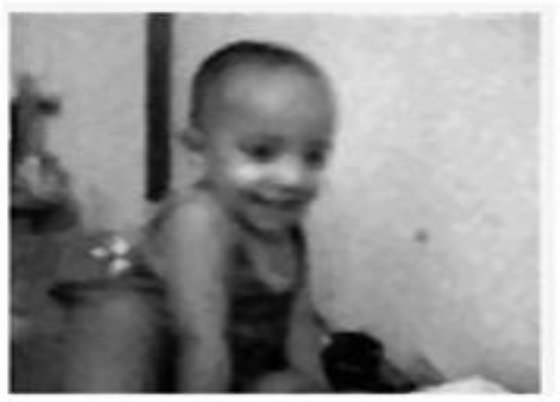

Figure 7c) HR image after IBP and image restoration
Table 1 indicates that Iterated Back Projection outperforms Non uniform Interpolation in its work and thus produce better results.

Table 1: PSNR Table Comparison

\begin{tabular}{|c|c|c|c|c|}
\hline \multicolumn{5}{|c|}{$\begin{array}{c}\text { PSNR Values of Output HR Images obtained from } \\
\text { different input image datasets }\end{array}$} \\
\hline $\begin{array}{c}\text { Test } \\
\text { image }\end{array}$ & NUI & IBP & $\begin{array}{c}\text { NUI } \\
\text { followed by } \\
\text { restoration }\end{array}$ & $\begin{array}{c}\text { IBP } \\
\text { followed by } \\
\text { restoration }\end{array}$ \\
\hline Crow & 25.67 & 29.44 & 32.575 & 38.98 \\
\hline baby1 & 23.34 & 27.45 & 30.764 & 33.67 \\
\hline baby2 & 22.25 & 27.54 & 30.864 & 32.259 \\
\hline baby3 & 19.69 & 25.93 & 31.452 & 32.45 \\
\hline Jackfruit & 22.88 & 27.65 & 29.652 & 33.065 \\
\hline Eye & 24.99 & 28.68 & 32.761 & 35.987 \\
\hline Portrait & 24.79 & 29.73 & 33.718 & 36.87 \\
\hline
\end{tabular}

\section{CONCLUSION \& FUTURE SCOPE}

The proposed method performs an efficient reconstruction of high resolution images from input multiple low resolution frames using two super resolution algorithms, non uniform interpolation and iterated back projection. Additional non redundant information from the registered low resolution images is acquired using the spatial domain method based on Taylor series. The obtained high resolution images from both super resolution approaches are enhanced using improved Wiener restoration and edges are sharpened using iterative median filter. Results from PSNR metric clearly indicate that iterative back projection method outperforms the non uniform interpolation approach. Restoration using improved Wiener filter requires no prior knowledge of the degradation, which makes it ideal for realistic applications. Moreover, since Wiener restoration is carried out iteratively, optimal mean square error is achieved. Finally iterative median filter removes and undo the effect of artifacts along the edges which further enhances the image and sharpens it.

Some of the output high resolution images exhibit halo effect even after restoration, which is not fully removed by the iterative median filter. A more efficient edge enhancement filter like bilateral filter may be employed for better results [32]-[33]. Also for better restoration of the degraded high resolution images, appropriate blur identification techniques may be applied. Researches in this direction are still open.

\section{REFERENCES}

[1] M.K Park S.C Park and M.G Kang. Super-resolution Image Reconstruction: A Technical Overview. IEEE Signal Processing Magazine, 20:21-36, May 2003.

[2] S. Borman and R.L. Stevenson, Super-resolution from image sequences: A review. Proceedings, Midwest Symp, Circuits and Systems, pp. 374-378, 1999.

[3] Sean Borman and Robert Stevenson, Spatial Resolution Enhance ment of Low Resolution Image Sequences - A 
Comprehensive Review with Directions for Future Research, Opt. Eng., July 1998.

[4] David Peter Capel, Image Mosaicing and SuperResolution. In Proceedings of the 10th International Conference on Pattern Recognition, Atlantic City, NJ, volume 2, pages 115-120,June1990.

[5] Daniel Glasner, Shai Bagon \& Michal Irani, Super Resolution from a Single Image, IEEE transactions, 2000

[6] S. Farsiu, D. Robinson, M. Elad, P. Milanfar. Fast and Robust multi frame super resolution, IEEE transactions on Image Processing, Vol 13, pp 1327 - 1344, 2004

[7] T. S. Huang and R. Y. Tsai, Multi-frame image restoration and registration, Advances in Computer Vision and Image Process., vol. 1, pp. 317-339, 1984.

[8] Patrick Vandewalle, Sabine Sstrunck and Martin Vetterli. Super Resolution Images Reconstructed from Aliased images, IEEE Transactions 1998

[9]Sanket.B. Kasturiwala, S.A.Ladakhe, A spatial domain Super-resolution approach for Soybean Leaf deceased image. vol.3, Issue 2, pp. 97-106, 2013.

[10] T.R Jones, W.T Freeman and E.C Pasztor, Example based super resolution. In IEEE Transactions on Computer Graphics and applications, volume 22, pages 56-65, April 2002.

[11] Kyaha Choi, Changhyun Kim and Jong Beom Ra, Example based superresolution via structure analysis of patches. In IEEE Signal Processing Letters, volume 20, pages 56-65, April 2013.

[12] D. Keren, S. Peleg and R, Brada, Image sequence enhancement using sub pixel displacements, Proceedings of IEEE Computer Society Conference On Computer Vision and Pattern Recognition (CVPR 88), pp 742-746, USA, June 1988

[13] M. Irani and S. Peleg, Improving resolution by image registration, In CVGIP: Graphical Models and Image Processing, volume 53, pages 231-239, May 1991.

[14] Capel.D and Zisserman.A, Computer vision applied to super-resolution. IEEE Signal Processing: Image Communication, 5, 511-526,2003

[15] Rajan D, Chaudhari Sand Joshi M V, Multi Objective super-resolution: Concepts and examples, IEEE Signal Processing Magazine, vol 20, pp 49-61, 2003.

[16] H. Stark and P. Oskoui, High resolution image recovery from image plane arrays, using convex projections, J. Opt. Soc. Am. A, vol. 6, pp. 1715-1726, 1989.

[17] C. Fan, J. Zhu, J. Gong, and C. Kuang. POCS superresolution sequence image reconstruction based on improvement approach of Keren registration method, in Proc. 6th Int. Conf. ISDA, pp 333-337, Oct 2006.

[18] G. Chantas, N. Galatsanos, and N. Woods., Superresolution based on fast registration and maximum a posteriori reconstruction, IEEE Trans. Image Process., vol. 16, no. 7, pp. 1821-1830, Jul. 2007.

[19] S. Belekos, N. Galatsanos, and A. Katsaggelos, Maximum a posteriori video super-resolution using a new multichannel image prior, IEEE Trans. Image Process., vol. 19, no. 6, pp. 1451-1464, June2010.
[20] H. Ji and C. Fermuller, Robust wavelet based superresolution reconstruction, Theory and algorithm, IEEE Trans, Pattern Analysis, Intell vol 31, no 4, pp 649-660, April 2009

[21] M. Irani \& S. Peleg. Motion analysis for image enhancement resolution, occlusion and image representation, Vol 4, pp 324 335, 1993.

[22] Carman Neustaedter. An Evaluation of Optical Flow using Lucas and Kanades algorithm, CVPR,2002.

[23] B. Marcel, M. Briot and R. Murrieta, Calcul de translation et rotation par la transformation de Fourier, Traitement du Signal, 14(2), pp 135- 149, 1997.

[24] L. Luchesse and M. Cortelazzo, A noise robust frequency domain technique for estimating planar roto-translation, IEEE transactions in Signal Processing, vol 48, pp 17691786, June 2000

[25] Martin A. Fischler, Robert C. Bolles, Random Sample Consensus: A paradigm for Model fitting with applications to Image analysis and automated cartography, Comm of the ACM, vol 24(6), pp 381-395, June 1981.

[26] D. Sun, S. Roth, and M J Black, Secrets of optical flow estimation and their principles, In IEEE International Conference on Computer Vision and Pattern Recognition, 2010.

[27] S. baker and T. Kanade, Super-resolution optical flow, Technical report, CMU, 1999

[28] Russel Hardie. A Fast Image Super-Resolution Algorithm Using an Adaptive Wiener Filter, IEEE Transactions On Image Processing, Vol 16, No 12 December 2007.

[29] Elad, M. and Feuer, A. Restoration of single superresolution image from several blurred, noisy and downsampled measured images. IEEE Trans- actions on Image Processing, Issuee 6, Vol 12, pp 164658, 1997.

[30] A. Cheref \& C. Serief. Deblurring And Denoising with Edge Enhancement of Satellite Images Using Super Resolution Tech- niques, 2006

[31] M. V. W. Zibetti \& J. Mayer. Outlier Robust and edge preserving simultaneous Super Resolution, In Proceedings of IEEE Interna tional Conference on Image Processing, pp 1741 - 1744, 2006

[32] C. Tomasi and R. Manduchi, Bilateral filtering for gray and color images, IEEE International Conference Computer Vision, New Delhi, India, pp 836-846, January 1998.

[33] M. Elad, On the Bilateral filter and ways to improve it, IEEE International Transactions Image Processing vol 11, pp 1141-1151, October 2002.

\section{AUTHOR'S PROFILE}

Nisha B R Obtained her BTech in Computer Science \& Engineering from Kerala University, Trivandrum, Kerala in 2006. She did her M.Tech in Image Processing from Cochin University of Science\& Technology, Kerala. She has over 4 years of teaching experience at FISAT, MG University, Kerala, India. 
Dr. Priya S Obtained her BTech in Computer Science \& Engineering from Kerala University, MTech in Computer Science \& Engineering from Pondicherry University, Pondicherry India and $\mathrm{PhD}$ in Information \& Communication Engineering from Anna University, Chennai, India. She is currently working as Associate Professor in the Department of Computer Science \& Engineering in Govt Model Engineering College, Thrikkakara, Ernakulam, Kerala, India. Her experience as a faculty is more than 18 years as of now. She is a life member of ISTE
Dr. Ashok kumar T. Obtained his BE in Electronics \& Telecommunication Engineering in 1994 , MTech in Electronics Design \& Technology from Indian Institute of Science (IISc), Bangalore, India ,MBA from IGNOU and PhD in Information\& Communication Engineering from Anna University, Chennai, India. His experience as a faculty is more than 20 years, teaching various courses in undergraduate as well as postgraduate level. He is working as an Associate Professor in the Department of Electronics Engineering in Govt. College of Engineering, Cherthala, Kerala, India. He is a life member of ISTE. 